\title{
ESTUDIO DE LA BIOSORCION DE ROJO ÁCIDO 18, AZUL BÁSICO 99 Y AMARILLO BÁSICO 57 PRESENTES EN LOS TINTES DE CABELLOS CON RESIDUOS DE HOJAS DE TÉ VERDE
}

\author{
Luciano Bellatin, Oscar Herrera ${ }^{1}$, Abel Navarro ${ }^{2}$, Rosario Sun-Kou ${ }^{3}$ y Bertha Llanos ${ }^{1 *}$
}

\begin{abstract}
RESUMEN
Se investigó el uso de hojas de té verde (TV) molidas con diámetro de partículas malla 30 y 40 de la serie Tyler, en la remoción de los colorantes Amarillo 57 (AM 57), Azul básico 99 (AZB 99) y Rojo ácido 18 (RA 18), a partir de soluciones acuosas a temperatura ambiente, mediante experimentos de desorción discontinuos. Los parámetros evaluados en la capacidad de adsorción fueron: $\mathrm{pH}$, masa de adsorbente, concentración de los colorantes, velocidad de adsorción y fuerza iónica, apoyados por los análisis de FTIR, SEM, EDX y TGA. Los resultados se han analizado usando los modelos matemáticos de Langmuir, Freundlich, Temkin, Dubinin-Radushkevich y Redlich-Peterson. Estos resultados muestran que la adsorción se da mediante un mecanismo mixto. Los parámetros determinados ponen en claro una alta afinidad del adsorbente con el AZ 99 con una máxima capacidad de adsorción de $287,2 \mathrm{mg} \mathrm{g}^{-1}$, seguido con el AM 57 con un $\mathrm{q}_{\text {máx }} 120 \mathrm{mg} \mathrm{g}^{-1}$ y con el RA 18 con q $_{\text {máx }}$ de $58 \mathrm{mg} \mathrm{g}^{-1}$, elucidando el potencial uso de este adsorbente para la remoción de los colorantes en soluciones acuosas mediante una cinética de pseudo-segundo orden, especialmente el AZB99. Altas concentraciones de la fuerza iónica disminuyen la adsorción de los tres colorantes.

Palabras clave: Hojas de té verde, Tintes de cabello, Amarillo básico 57, Azul básico 99 y Rojo ácido 18.
\end{abstract}

\begin{abstract}
The use of waste green tea leaves (TV) with particle diameter 30 and 40 of the Tyler series, on the removal of the dyes basic yellow 57 (AM 57), basic blue 99 (AZB 99) and acid red 18 was investigated from aqueous solutions at room temperature by intermittent desorption experiments. The parameters evaluated in the adsorption capacity were: $\mathrm{pH}$, mass of adsorbent, the concentration of dye adsorption rate and ionic strength, supported by the FTIR, SEM, EDX and TGA analysis. The results are analyzed using mathematical models of Langmuir, Freundlich, Temkin, Dubinin- Radushkevich and Redlich -Peterson. The results show that the adsorption is given by a mixed mechanism. The determined parameters show a high affinity of the adsorbent with AZ 99 with a maximum adsorption capacity of $287.2 \mathrm{mg} \mathrm{g}^{-1}$, followed with by AM $57120 \mathrm{mg} \mathrm{g}^{-1}$ and the RA 18 with $58 \mathrm{mg} \mathrm{g}^{-1}$ elucidating the potential use of the adsorbent for the removal of the dyes in aqueous solutions by pseudo- second order kinetic. High concentration of the ionic strength decreases the adsorption of the three dyes.

Keywords: Green tea leaves, hair dyes, basic Yellow 57, Basic blue 99 and acid Red 18.

\footnotetext{
1* Departamento de Ciencias Exactas - Sección Química, Facultad de Ciencias, Universidad Peruana Cayetano Heredia. Av. Honorio Delgado 430 - Urb. Ingeniería SMP Lima 31 - Perú.

2 Science Department, Borough of Manhattan Community College New York, NY, USA. Católica del Perú, Av. Universitaria 1801 - Lima 32

bertha.1lanos@upch.pe.
}

3 Departamento de Ciencias -Sección Química, Facultad de Ciencias e Ingeniería Pontificia Universidad
\end{abstract}




\section{INTRODUCCIÓN}

El cabello no tiene función vital en el cuerpo humano pero provee un signo externo de salud y comunicación social $^{1}$. El cabello humano contiene $85 \%$ proteína, $7 \%$ agua, 3\% lípidos, 4,7\% proteínas sulfuradas y trazas de minerales (i.e. hierro, cinc y cobre) ${ }^{2}$.

El uso de tintes de cabello se remonta desde hace 4000 años atrás. En la época del Imperio Romano, peines de plomo eran inmersos en vinagre y pasados por los cabellos para oscurecer las canas ${ }^{1,3}$. Hoy, millones de consumidores usan tintes de cabello. Teniendo en cuenta la extensión y frecuencia del contacto humano con productos de cabello, la demanda de sus ingredientes han aumentado y por ende los desechos asociados a ellos. El hallazgo de que la piel humana no es una barrera impermeable para algunas sustancias inició la investigación de adsorción de tintes de cabello y de sus ingredientes ${ }^{4}$. Publicaciones recientes vinculadas con los daños de estos tintes incluyen: (i) Un estudio realizado por la US Food and Drug Association (FDA) encontró que el acetato de plomo (el ingrediente activo de productos oscurecedores graduales empleado en la fórmula Grecian) es potencialmente tóxico. (ii) Artículos que vinculan el desarrollo de algunos tipos de cáncer (leucemia, linfoma noHodgkin, cáncer de vesícula, cáncer de sangre) con el uso de tintes de cabello. (iii) Recientemente, un conocido carcinógeno humano, 4-ABP, fue encontrado en algunos tintes de cabello. En los Estados Unidos la seguridad de los cosméticos, incluyendo el de los tintes de cabello, está bajo la responsabilidad legal de la US Food and Drug Association (FDA). Por lo expuesto, algunos ingredientes de tintes de cabello aún no han demostrado ser totalmente inocuos para el uso humano y su producción industrial y los residuos que acarrean podrían crear contaminación en varios ecosistemas. Con respecto al medio ambiente, la presencia de tintes solubles en agua, proporciona tonalidades al medio que lo alejan de la idea de pureza. Si bien su toxicología no es tan nociva como la presencia de metales pesados en solución acuosa, pero el simple hecho de otorgar un color no natural al agua, la convierte en prioridad de tratamiento. Además, la presencia de sustancias coloreadas en sistemas acuíferos altera la biota, ya que estas moléculas absorben la radiación solar (vital para la fotosíntesis) y privan de esa energía a las plantas acuáticas (fitoplancton en mares) y otros microorganismos que requieren el uso de la energía solar para su supervivencia. Si se combinan ambos efectos de los tintes de cabello (efecto carcinógeno y color en el agua) en la naturaleza podríamos estar enfrentando un problema de bio-magnificación, dado que a la fecha se desconoce la penetración de estas moléculas coloreadas en los tejidos vegetales y animales (por ejemplo de peces), los cuales mediante la cadena trófica es incorporada en la dieta humana. Afortunadamente, con el propósito de mantener el agua potable dentro de los límites permisibles para su consumo, diferentes métodos de purificación han sido desarrollados para su tratamiento. La adsorción con carbón activado es el método comúnmente usado para remover tintes, pero debido a su alto costo se sigue en la búsqueda de materiales más baratos con similar o mayor eficiencia ${ }^{5}$. La biorremediación es una alternativa de remoción de tintes; la biosorción ofrece las ventajas de operación a bajo costo, reducción del volumen de lodos químicos y biológicos, además de que sólo utiliza materia muerta proveniente de microorganismos. Este proceso también permite la regeneración del biosorbente y la recuperación del adsorbato ${ }^{6,7}$.

Ante estos problemas, primero, el posible efecto carcinógeno de los tintes en humanos, segundo, la contaminación de las aguas residuales generada por las industrias que usan tintes como materias primas y tercero, la ineficiencia y alto costo de las técnicas convencionales de purificación de aguas, se justifica la búsqueda de tecnologías limpias con nuevos y/o mejorados adsorbentes alternativos. 


\section{Acerca del adsorbente}

El té verde es originario de Asia y es mundialmente conocido por sus propiedades medicinales y antioxidantes. En las últimas décadas del siglo pasado, el té verde retomó una importancia por sus propiedades curativas y analgésicas. De acuerdo a su composición química, las hojas de té verde contienen mucha fibra (carbohidratos insolubles en agua) y polifenoles (catequina, epicatequina, galocatequina, epilogalocatequinas-3-galato y otros) (figura 1). Estos grupos funcionales presentes en las hojas de té verde son de vital importancia para la adsorción, ya que la presencia de fibras en su estructura promueve la absorción de agua y por lo tanto aumenta la superficie específica para hacer más accesibles los centros de adsorción (i.e. polifenoles) para los contaminantes. En la actualidad no hay resultados reportados en la retención de los contaminantes con té verde, excepto un estudio con cromo ${ }^{8}$, con resultados de la adsorción casi despreciables.<smiles>O=C(OC1Cc2c(O)cc(O)cc2OC1c1cc(O)c(O)c(O)c1)c1cc(O)c(O)c(O)c1</smiles><smiles>Oc1cc(O)c2c(c1)OC(c1ccc(O)c(O)c1)C(O)C2</smiles>

Epilogalocatequina-3-galato

Figura 1: Estructura de algunos fenoles presentes en las hojas de té verde

\section{Acerca del adsorbato}

Nohynek et al. ${ }^{1}$, mencionan cuatro tipos de tintes de cabello: permanentes, semipermanentes, sales metálicas y tintes naturales. Entre ellos, uno de las más importantes son los del tipo semipermanente que están presentes en casi todas las formulaciones de los tintes que se encuentran en el mercado, porque le otorgan las combinaciones de color necesarias para alcanzar las tonalidades de cabello requeridas por los usuarios. Los tintes semipermanentes, a su vez, se sub-clasifican en tintes aniónicos y catiónicos. Por eso se seleccionó a los tres colorantes: Amarillo básico 57, Azul básico 99, tintes catiónicos, y Rojo ácido 18 (tinte aniónico) presentes en los tintes, y fueron seleccionados en base a: su frecuente uso en la formulación de tintes, su solubilidad en agua y su fácil determinación por espectrofotometría UV-visible ${ }^{9,10}$. Las estructuras de los tintes se muestra en la figura 2; se aprecia zonas muy polares y no polares por lo que existe una alta afinidad del adsorbente hacia los colorantes. 


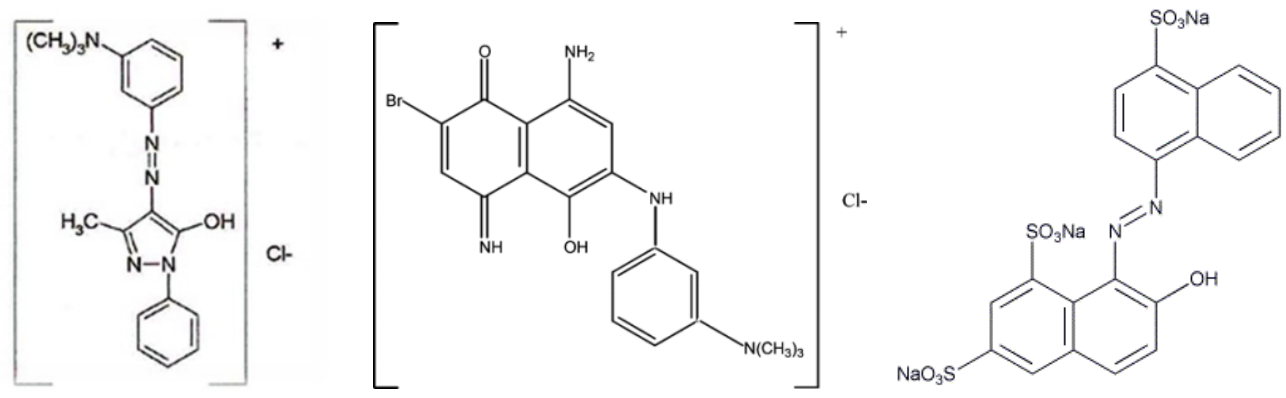

Figura 2: Estructura de los colorantes: Amarillo básico 57 (izquierda), Azul básico 99 (centro) y Rojo ácido 18 (derecha).

\section{Sobre el proceso de adsorción}

La adsorción es el fenómeno de atracción de sustancias (gas o líquido) que se encuentran en una determinada fase, hacia la superficie de un sólido. La adsorción es un fenómeno espontáneo debido a la existencia de fuerzas no compensadas en la interfase sólido-fluido (línea de división entre fases). Adicionalmente, en el campo de la retención y eliminación de contaminantes, el uso de isotermas de adsorción es ampliamente difundido, así como, la determinación del parámetro "q" que mide la capacidad de adsorción del adsorbato sobre la superficie del sólido, expresado en mg de adsorbato/g de adsorbente ${ }^{11}$. Las isotermas más usadas son: Isoterma de Langmuir ${ }^{1,12}$, Isoterma de Freundlich ${ }^{1,13}$.

El mecanismo de adsorción se describe como una serie de etapas consecutivas para el transporte del adsorbato desde la solución hasta su posición final en el sitio activo ${ }^{14}$. En biosorción, los modelos cinéticos matemáticos más utilizados son: pseudo-primer orden o ecuación de Lagergren ${ }^{15}$, pseudo-segundo orden de Ho y McKay ${ }^{16}$.

\section{Materiales y métodos}

\section{PARTE EXPERIMENTAL}

\section{Reactivos}

Los reactivos usados en la investigación son los siguientes: Té verde puro a granel "Schagreen", producido por Alprosur, La Convención, Cusco, Perú; colorante aniónico rojo ácido 18 (75\% pureza, Sigma Aldrich); colorante catiónico amarillo básico 57 (99\% pureza, Vanshi Chemical); colorante catiónico azul básico 99. (90\% pureza, Vanshi Chemical); ácido clorhídrico concentrado PA, Merck; hidróxido de sodio (PA, Merck) y azul de metileno (Merk)

\section{Preparación de los adsorbentes}

Se pesó $90 \mathrm{~g}$ de té verde (TV) y se colocó equitativamente en dos vasos de precipitado de $2 \mathrm{~L}$ y se agregó a cada uno 1,5 L de agua potable recién hervida. Con agitación ocasional por media hora, se mantuvo los vasos de precipitado en calentamiento sin exceder $\operatorname{los} 90{ }^{\circ} \mathrm{C}$, y posteriormente se filtró la mezcla. Se repitió varias veces el procedimiento anterior hasta que el agua de extracción quedó de un tenue color amarillo-verdoso. Una vez terminada la extracción, el residuo de las hojas de té verde fue filtrado al vacío, luego fue secado en estufa a $50^{\circ} \mathrm{C}$ por $48 \mathrm{~h}$. Luego se molió mediante un molino Cole Parmer y se pasó a través de la serie 
de tamices con números de malla: 20,30 y 40 por 10 minutos mediante el uso de un tamizador de vibración marca Retsch. Las partículas de mayor tamaño retenidas por la malla 20, se volvieron a moler y tamizar. Para los estudios de adsorción se utilizó $70 \mathrm{~g}$ de cada una de las fracciones de las mallas 30 y 40 (con tamaño entre 425 y $850 \mu \mathrm{m}$ ). Se homogeneizó la mezcla, se almacenó en frascos de plástico cerrados y se guardó dentro de un desecador con sílica gel.

\section{Preparación de las soluciones}

Previo a la adsorción, los colorantes rojo ácido 18, amarillo básico 57 y azul básico 99 se secaron en una estufa a $50^{\circ} \mathrm{C}$ por 24 horas. Luego se preparó soluciones stock de $1000 \mathrm{mg} / \mathrm{L}$ con agua destilada. A partir de cada una de ellas, se preparó soluciones diluidas de diferentes concentraciones necesarias para el estudio.

\section{Análisis de las soluciones}

La cuantificación de las soluciones acuosas de los diferentes colorantes se llevó a cabo mediante espectrofotometría UV-visible (UV Mini 1240 Shimatzu). Los colorantes fueron cuantificados a $\lambda=507,380$ y $574 \mathrm{~nm}$, respectivamente.

\section{Caracterización de los adsorbentes}

Los espectros de infrarrojo fueron obtenidos por espectroscopía infrarrojo con transformada de Fourier, usando el espectrofotómetro Perkin Elmer Spectrum 100. Los análisis termogravimétricos (TGA) se realizaron usando el equipo Shimadzu model 50; el método consiste en registrar la pérdida de peso de la muestra durante el incremento de la temperatura; se escaneó a una velocidad de calentamiento de $10{ }^{\circ} \mathrm{C} / \mathrm{min}$ bajo flujo de nitrógeno desde temperatura ambiente hasta $700{ }^{\circ} \mathrm{C}$. El análisis morfológico se realizó en un microscopio electrónico de barrido FEI Quanta 200; SEM y EDX.

\section{Experimentos de adsorción y desorción}

\section{Efecto del pH}

Se preparó una solución de colorante de una determinada concentración a partir de una dilución del stock. Se secó el adsorbente (TV) a $60^{\circ} \mathrm{C}$ por dos horas previas y se pesó en varios Erlenmeyer una determinada masa de adsorbente y sobre ellos se agregó $50 \mathrm{~mL}$ de la solución de colorante con el $\mathrm{pH}$ correspondiente ajustado mediante soluciones diluidas de $\mathrm{HCl}$ e $\mathrm{NaOH}$. También se preparó blancos, que consistieron en la mezcla de té verde con agua destilada al pH correspondiente. Las muestras se agitaron a $250 \mathrm{rpm}$ en un agitador orbital Innova modelo 2100, por 48 horas. Se filtró las mezclas y se midió las concentraciones mediante el espectrofotómetro UV-visible Shimadsu. Se midió asimismo el pH final del filtrado.

\section{Parámetros de equilibrio termodinámico}

Se preparó una solución de colorante de una determinada concentración a partir de una dilución del stock y con la adición de solución de $\mathrm{HCl}$ se llevó al pH óptimo luego se virtió 50 $\mathrm{mL}$ a varios Erlenmeyer que contenían distintas masas: 20, 30, 50, 75 y $100 \mathrm{mg}$, previamente desecadas a $60^{\circ} \mathrm{C}$. Las muestras se agitaron a $250 \mathrm{rpm}$ por 48 horas. La cuantificación se hizo igual que para el $\mathrm{pH}$.

Para obtener las isotermas de adsorción, se pesó una cantidad de adsorbente y a cada uno ellos se adicionaron $50 \mathrm{~mL}$ de cada una de las soluciones de distintas concentraciones ajustadas al pH óptimo y procedió de la misma manera que para el efecto de $\mathrm{pH}$ y masa. Teniendo los valores de Capacidad de Adsorción (mg.g $\mathrm{g}^{-1}$ ) y concentración en el equilibrio (mg. $\mathrm{L}^{-1}$ ) del colorante, se procedió al modelamiento de acuerdo a las isotermas de Langmuir, Freundlich, Temkin, Dubinin-Radushkevich y Redlich-Peterson. 


\section{Cinética de adsorción}

Se preparó $1 \mathrm{~L}$ de una solución de colorante a partir de una dilución del stock y con la adición de $\mathrm{HCl}$ concentrado, se llevó al pH óptimo; se dejó en agitación constante evitando la turbulencia y se adicionó rápidamente una cantidad de adsorbente seco (correspondiente a la proporción masa óptima) de tal que siempre el adsorbente este suspendido en la solución e inmediatamente se accionó el cronómetro. Cada cierto tiempo se tomó alícuota (alrededor de 8 $\mathrm{mL}$ ), se filtró y analizó el contenido de colorante. El procedimiento se siguió durante 48 horas. Con los datos se procedió al modelamiento de acuerdo a los modelos de pseudo-primer orden, pseudo-segundo orden y Elovich.

\section{Fuerza iónica}

Se probó el efecto de tres sales solubles en agua: Cloruro de sodio, nitrato de sodio y nitrato de calcio; en diferentes concentraciones: $0,01 \mathrm{M} ; 0,05 \mathrm{M}$; 0,1 M y 0,5 M. Se pesó las cantidades de sal necesaria para preparar las soluciones a las concentraciones requeridas. Se preparó una solución de colorante a partir de una dilución del stock y con la adición de $\mathrm{HCl}$, se ajustó al pH óptimo. En esta solución se disolvió las sales previamente pesadas. Se agregó $50 \mathrm{~mL}$ de estas soluciones a una determinada masa de TV seco. Las muestras se agitaron a $250 \mathrm{rpm}$ y por 48 horas. Para su cuantificación se llevó a cabo el mismo procedimiento que para los otros estudios.

\section{RESULTADOS Y DISCUSIÓN}

\section{Caracterización de los adsorbentes}

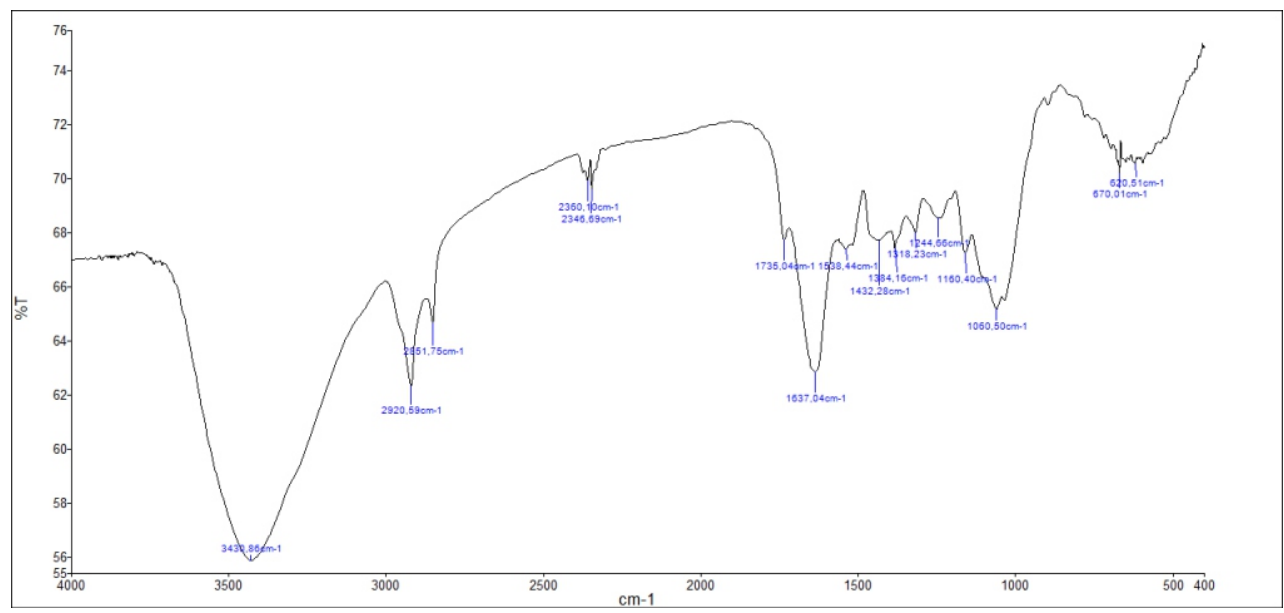

Figura 3. Espectro FTIR de las hojas té verde (TV).

En el espectro del té verde (figura 3) se muestra una señal a $3430 \mathrm{~cm}^{-1}$ atribuido al estiramiento $\mathrm{O}-\mathrm{H}$ (aldehído, alcohol, agua); a $2921 \mathrm{~cm}^{-1}$ se le asigna al estiramiento $\mathrm{C}-\mathrm{H}$ (alquilo), acompañado de una señal débil que se debe a $\mathrm{C}=\mathrm{C}-\mathrm{H}$; aproximadamente a $1735 \mathrm{~cm}^{-1}$ se le atribuye al estiramiento $\mathrm{C}=\mathrm{C}$ (anillo aromático, carbohidratos) posiblemente proveniente de 
ligninas; la señal a $1637 \mathrm{~cm}^{-1}$ se asigna a grupos carbonilo $\mathrm{C}=\mathrm{O}$; la $1432 \mathrm{~cm}^{-1}$ se asocia a la vibración de tensión -C-O-C-, y la $1050 \mathrm{~cm}^{-1}$ se atribuye a la deformación en el plano aromático $=\mathrm{C}-\mathrm{H}$ (benceno).

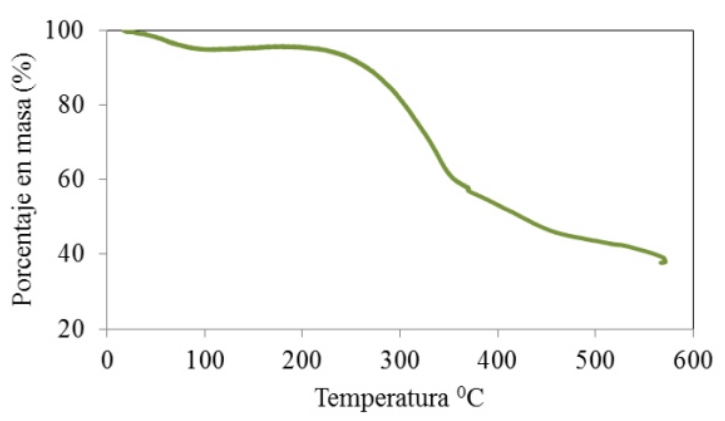

Figura 4. Análisis termogravimétrico del té verde (TV)

En el análisis termogravimétrico (TGA) (figura 4) se aprecia que el TV se degrada con la temperatura un dos etapas; hay una pequeña pérdida de peso alrededor de $100{ }^{\circ} \mathrm{C}$, entre $100 \mathrm{y}$ los $200{ }^{\circ} \mathrm{C}$ la muestra no sufre cambio y de ahí en adelante tiene la mayor pérdida de peso; y no es estable a temperaturas encima de $200{ }^{\circ} \mathrm{C}$. La superficie específica de las hojas de té verde con diámetro de partículas comprendidas entre las mallas 30 y 40 de la serie de Tyler (425$850 \mu \mathrm{m})$ según el método de azul de metileno es de $423,4 \mathrm{~m}^{2} \mathrm{~g}^{-1}$, está área es ligeramente menor a la del carbón activado que oscila entre $500-1500 \mathrm{~m}^{2} \mathrm{~g}^{-1}$ dependiendo del diámetro de las partículas.

En la figura 5, se tiene la microscopía (MEB) a $800 \mathrm{X}$ donde se aprecia la estructura porosa y laminar y en el análisis EDX del adsorbente se tiene que más del $97 \%$ es de carbono y oxígeno; confirmando esto que los insolubles del té verde son polifenoles y celulosa y también tiene $2,24 \%$ de calcio insoluble como carbonato que queda adherida a la superficie, microscopia de la izquierda a $400 \mathrm{X}$.
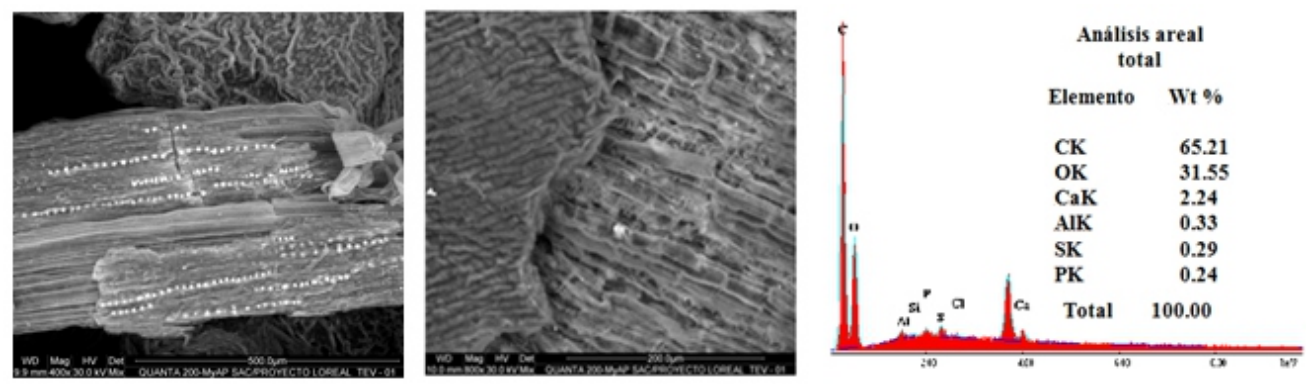

Figura 5. Micrografía MEB a 400X y 800 X y análisis EDX de té verde (TV) 
En la figura 6 se observa capacidad de adsorción del TV con los colorantes amarillo básico 57 (AM 57), azul básico 99 (AZB 99) y rojo ácido 18 (RA 18) en función de pH; los tres colorantes tienen la mayor capacidad de adsorción a distintos $\mathrm{pH}$ y considerando a los mismos como los óptimos para cada colorante; siendo estos pH 2 (RA 18), pH 4 (AM 57) y pH 4,5 (AZB 99); a pH mayores a los óptimos disminuye la adsorción haciéndose casi asintótica para el AM 57; en cambio para los otros colorantes hay una mayor disminución, especialmente en rojo ácido que no hay adsorción si el pH es igual o mayor a 4.

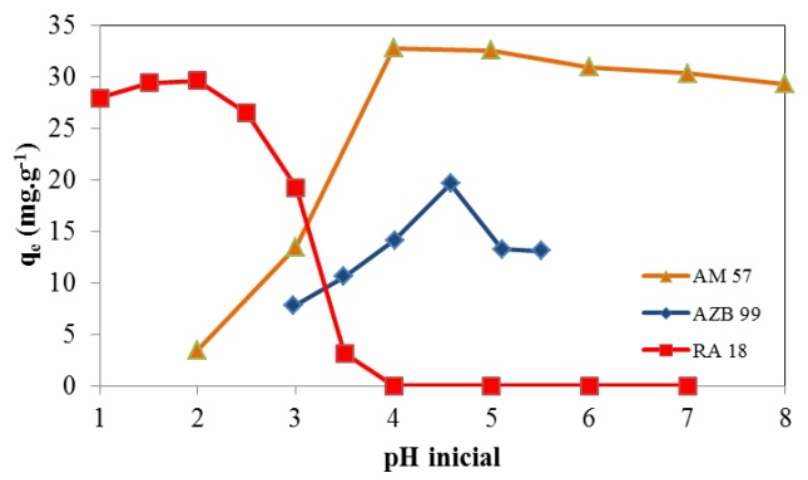

Figura 6. Efecto del pH en la adsorción de AM 57, AZB 99 Y RA 18 con TV a T ambiente

En la figura 7, se observa el efecto de la masa de TV con los tres colorantes en la capacidad de adsorción y es mejor con menor cantidad de adsorbente, especialmente para el azul con poquísima masa se tiene casi el $95 \%$ de adsorción, en cambio para el amarillo y rojo se requiere una mayor cantidad de adsorbente, pero siempre menor a los $100 \mathrm{mg}$ de adsorbente y este resultado es lo esperado usar poco cantidad de adsorbente pero con un buen porcentaje de adsorción.

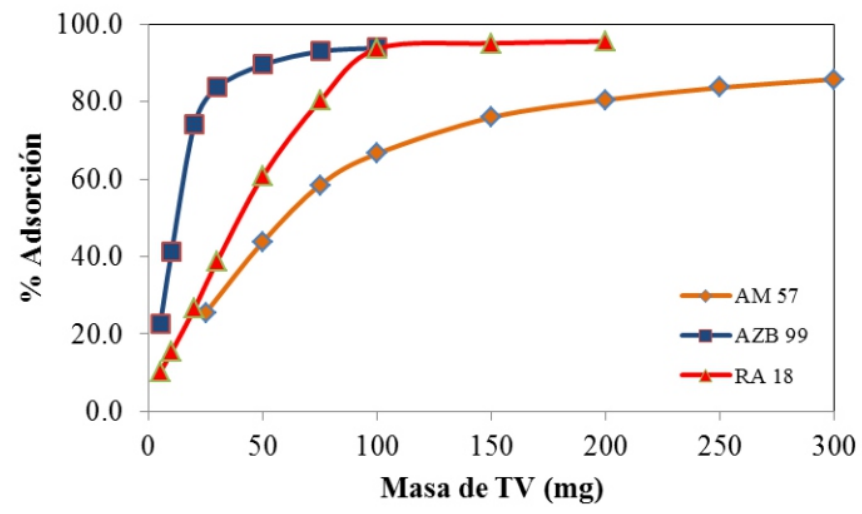

Figura 7. Efecto de la masa en la adsorción de AM 57, AZB 99 Y RA 18 con TV a T ambiente 
Tabla 1. Parámetros de adsorción de los colorantes AM 57, AZB 99 y RA 18 con té verde (TV) según las isotermas de Langmuir, Freundlich, Dubinin-Radushkevich, Temkin

y Redlich-Peterson

\begin{tabular}{|c|c|c|c|c|c|}
\hline \multicolumn{6}{|c|}{ Isoterma de Langmuir } \\
\hline Isoterma & $\mathrm{R}^{2}$ & $1 / q \max$ & $1 / q_{\max } \cdot K_{L}$ & $q_{\max }\left(\mathrm{mg} \mathrm{g}^{-1}\right)$ & $K_{L}\left(L_{m g}^{-1}\right)$ \\
\hline AM 57 & 0,9759 & 0,0050 & 0,3924 & 199,6 & 0,01 \\
\hline AZB 99 & 0,9823 & 0,0035 & 0,0727 & 287,2 & 0,05 \\
\hline RA 18 & 0,9988 & 0,0172 & 0,0299 & 58,0 & 0,58 \\
\hline \multicolumn{6}{|c|}{ Isoterma de Freundlich } \\
\hline Isoterma & $\mathbf{R}^{2}$ & $1 / \mathrm{nF}$ & $\log K_{F}$ & $\mathbf{n}_{\mathbf{F}}$ & $\mathrm{mg} \mathrm{g}^{-1} \begin{array}{l}\mathrm{K}_{\mathrm{F}} \\
\left(\mathrm{L} \mathrm{mg}^{-1}\right)\end{array}$ \\
\hline AM 57 & 0,9748 & 0,7575 & 0,5454 & 1,3 & 3,5 \\
\hline AZB 99 & 0,9880 & 0,7069 & 1,1548 & 1,4 & 14,3 \\
\hline RA 18 & 0,9569 & 0,0916 & 1,5778 & 10,9 & 37,8 \\
\hline \multicolumn{6}{|c|}{ Isoterma de Temkin } \\
\hline Isoterma & $\mathbf{R}^{2}$ & $\mathbf{R T} / \mathbf{b}_{\mathrm{T}}$ & $\left(\mathrm{RT} / \mathbf{b}_{\mathrm{T}}\right) \ln \mathrm{A}$ & $\mathbf{b}_{\mathrm{T}}$ & $A\left(L^{-1}\right)$ \\
\hline AM 57 & 0,8997 & 28,5253 & $-28,5253$ & 85,4 & 0,37 \\
\hline AZB 99 & 0,8647 & 39,1248 & 23,4455 & 62,3 & 1,82 \\
\hline RA 18 & 0,9823 & 3,9575 & 38,9260 & 615,9 & $1,88 \mathrm{E} 04$ \\
\hline \multicolumn{6}{|c|}{ Isoterma de Dubinin-Radushkevich } \\
\hline Isoterma & $\mathbf{R}^{2}$ & $-B_{\mathrm{DR}}$ & $\ln \mathbf{q}_{\mathrm{s}}$ & $\mathbf{B}_{\mathrm{DR}}$ & $\mathrm{q}_{\mathrm{s}}\left(\mathrm{mg} \mathrm{g}^{-}\right.$ \\
\hline AM 57 & 0,8304 & $-3,423 \mathrm{E}-07$ & 6,1980 & $3,43 \mathrm{E}-07$ & 491,79 \\
\hline AZB 99 & 0,5829 & $-1,455 \mathrm{E}-08$ & 4,4970 & $1,46 \mathrm{E}-08$ & 89,75 \\
\hline RA 18 & 0,7549 & $-2,0 \mathrm{E}-08$ & 3,9567 & $2,0 \mathrm{E}-08$ & 52,28 \\
\hline \multicolumn{6}{|c|}{ Isoterma de Redlich-Peterson } \\
\hline Isoterma & $\mathbf{R}^{2}$ & $\boldsymbol{\alpha}_{\mathrm{RP}} / \mathbf{K}_{\mathrm{RP}}$ & B & $\boldsymbol{\lambda}_{\mathrm{RP}}\left(\mathrm{L} \mathrm{mg}^{-1}\right)^{\beta}$ & $\mathrm{K}_{\mathrm{RP}}\left(\mathrm{L} \mathrm{g}^{-1}\right)$ \\
\hline AM 57 & 0,9789 & 0,0104 & 0,9 & 0,019 & 2,72 \\
\hline AZB 99 & 0,9905 & 0,0087 & 0,9 & 0,10 & 16,65 \\
\hline RA 18 & 0,9999 & 0,0280 & 0,9 & $-3,9$ & $-139,67$ \\
\hline
\end{tabular}

En la figura 8, se aprecia la tendencia de la adsorción del adsorbente TV con los tres colorantes; en el caso del AM 57 y del AZB 99 no se alcanza un máximo en las concentraciones de equilibrio, pero tienen la misma tendencia, solo que para el AZB 99 el TV tiene una mejor capacidad de adsorción; en cambio, con el RA 18 se aprecia un máximo y se alcanza rápidamente el equilibrio de adsorción pero no es tan buena como la adsorción del AZB 99. 
Los modelos matemáticos de Langmuir y Freundlich para el adsorbente con los tres colorantes tienen un alto coeficiente de linealidad, tal como se aprecia en la tabla 1, la máxima capacidad de adsorción según el modelo de Langmuir es de 287,6 $\mathrm{mg} \mathrm{g}^{-1}$ para el AZB 99 el que nos indica que este colorante se adsorbe mejor que los otros colorantes, aunque sus valores de $\mathrm{q}_{\text {máx }}$ también son bastantes aceptables.

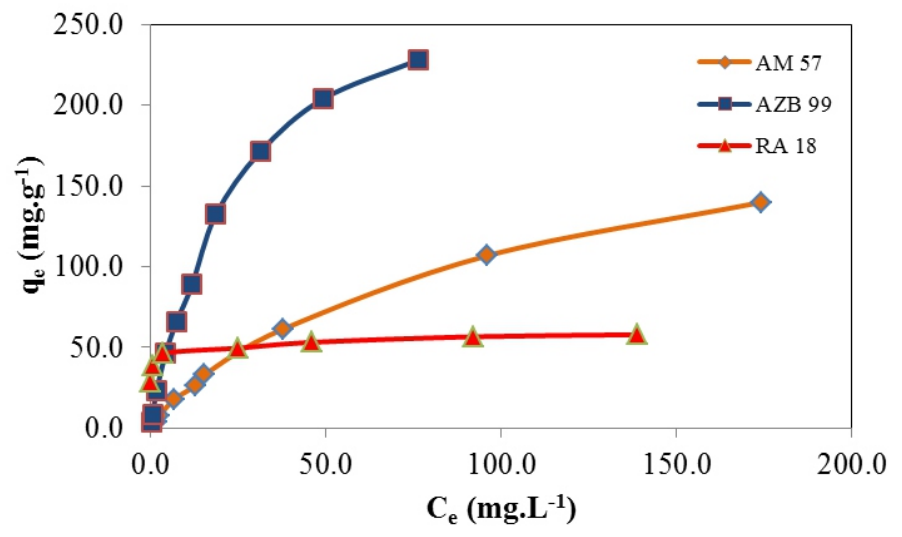

Figura 8: Adsorción de AM57, AZB99 y RA18 con 50 mg de TV a pH óptimos y $\mathrm{T}$ ambiente

Según Freundlich el que tiene una mejor constante $\mathrm{K}_{\mathrm{f}}$ es con el colorante RA 18. Estos resultados inducen a elucidar que los centros activos del adsorbente (TV) son equivalentes restringiéndose la adsorción en monocapa y no existe interacciones laterales entre las moléculas de los adsorbatos y la superficie es energéticamente heterogénea; esto se corrobora por los resultados obtenidos según el modelo matemático de Temkin ver (tabla 1). Los resultados obtenidos según el modelo de Dubinin-Radushkevich, implican que el adsorbente no tiene una estructura porosa. Según Redlich-Peterson el comportamiento del adsorbente se puede estimar sólo con el modelo de Langmuir, porque para la adsorción de los tres colorantes los valores de $\beta$ son 0,9 y muy cercanos a 1,0 y con buenos coeficientes de determinación (ver tabla 1).

En la figura 9 se presenta la cinética de adsorción de los tres colorantes con el té verde; con AZB 99 y RA 18 se alcanza el equilibrio de adsorción en 14 horas mientras que con AM 57 en tan solo 4 horas. El modelo matemático al que se ajustan con un alto coeficiente de determinación tal como se observa en la tabla 2 es de un pseudo-segundo orden pero con constantes de velocidad bajos para los primeros. 


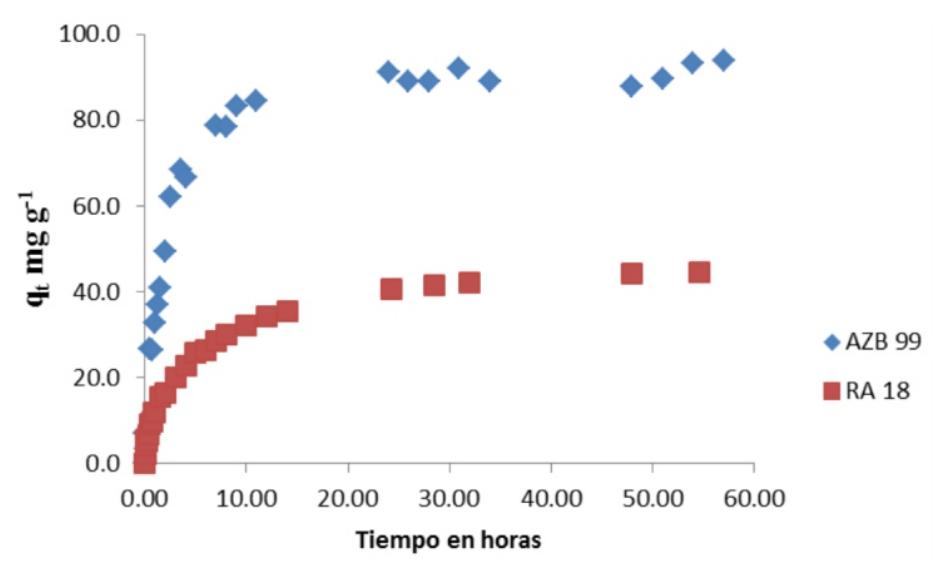

Figura 9. Cinética de adsorción de AM 57, AZB 99 y RA 18 con TV a pH óptimos y $\mathrm{T}$ ambiente

Según los coeficientes de determinación obtenidos para el modelo de Elovich, que están alrededor de 0,9 se puede elucidar que la adsorción entre el té verde y los tres colorantes no es una quimisorción ${ }^{17}$.

Tabla 2. Resultados de la cinética de adsorción de AM57, AZB99 y RA18 con TV

\begin{tabular}{|c|c|c|c|c|}
\hline \multicolumn{5}{|c|}{ MODELO DE PSEUDO-PRIMER ORDEN } \\
\hline Colorante & $\mathbf{R}^{2}$ & $-k_{1}$ & In qe & $k_{1}\left(h^{-1}\right)$ \\
\hline AM 57 & 0,7107 & $-0,1012$ & 2,3724 & 0,1012 \\
\hline AZB 99 & 0,7089 & $-0,0653$ & 3,7768 & 0,0653 \\
\hline RA 18 & 0,9761 & $-0,0908$ & 3,5686 & 0,0908 \\
\hline \multicolumn{5}{|c|}{ MODELO DE PSEUDO-SEGUNDO ORDEN } \\
\hline Colorante & $\mathbf{R}^{2}$ & $1 / q e$ & $1 / k_{2} q_{e^{2}}$ & $k_{2}\left(g g^{-1} h^{-1}\right)$ \\
\hline AM 57 & 0,9998 & $-0,0293$ & 0,0090 & 0,0954 \\
\hline AZB 99 & 0,9986 & 0,0107 & 0,0146 & 0,0078 \\
\hline RA 18 & 0,9932 & 0,0218 & 0,0611 & 0,0078 \\
\hline \multicolumn{5}{|c|}{ MODELO DE ELOVICH } \\
\hline Colorante & $\mathbf{R}^{2}$ & $(1 / \beta) \ln (\alpha \beta)$ & $\beta\left(\right.$ g.mg $\left.{ }^{-1}\right)$ & $\alpha\left(\mathrm{mg} \mathrm{g}^{-1} h^{-1}\right)$ \\
\hline AM 57 & 0,9228 & 24,279 & 0,3016 & 5019,59 \\
\hline AZB 99 & 0,9156 & 46,683 & 0,0829 & 578,02 \\
\hline RA 18 & 0,9435 & 16,525 & 0,1549 & 83,45 \\
\hline
\end{tabular}


En las figuras 10 y 11 se tiene los análisis por EDX; se aprecian que, efectivamente, sobre la superficie del adsorbente la adsorción de los colorantes, en el caso del AZB99 se tiene contenido de nitrógeno y bromo y en el RA18 también se tiene la presencia de nitrógeno y azufre, que son parte de la estructura de ambos colorantes (figura 2). Según la micrografía, el aspecto morfológico del TV no ha sufrido alteración comparada con el MEB de la figura 5.
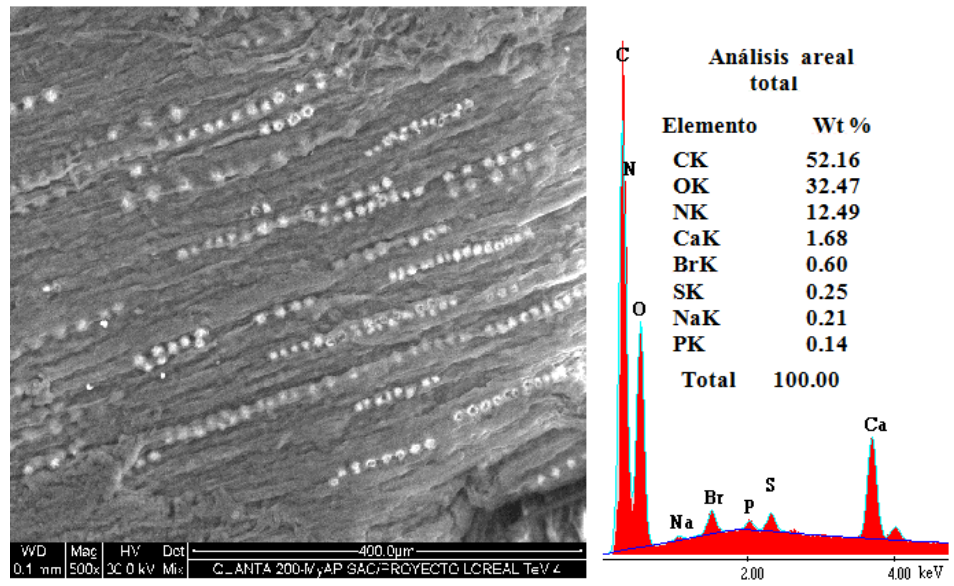

Figura 10. Micrografía MBE y análisis EDX de TV después de que ha adsorbido AZB 99
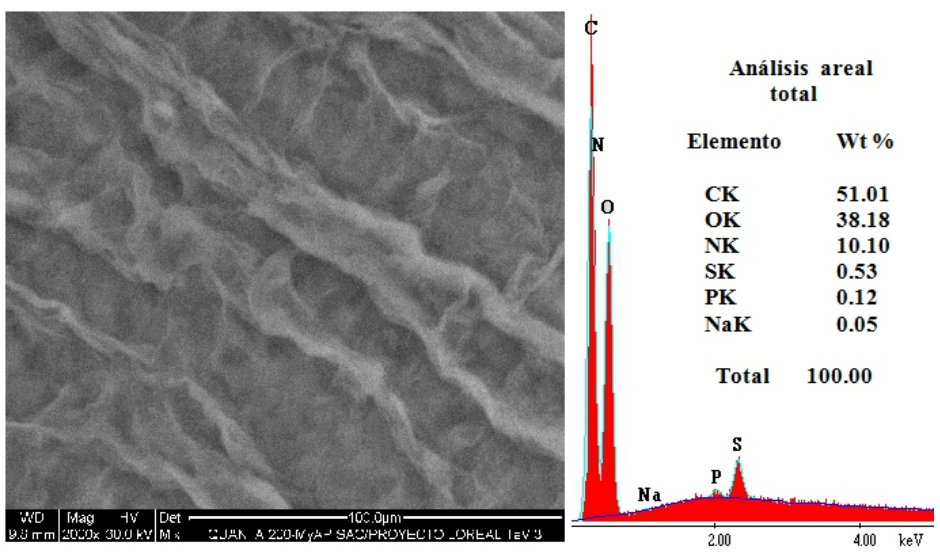

Figura 11. Micrografía MBE y análisis EDX de TV después de que ha adsorbido RA 18 
En las figuras 12, 13, 14 se muestran el efecto de la fuerza iónica en la adsorción de cada uno de los tres colorantes con el TV; la presencia de iones disminuye la capacidad de adsorción de los tres colorantes y si éstos están en mayor cantidad el efecto es mucho mayor; el tamaño de los iones también afecta en la adsorción si se compara ion nitrato y ion cloruro; también afecta la carga del ion $\mathrm{Ca}^{+2}$ comparada con el ion $\mathrm{Na}^{+}$; mayor deficiencia de electrones en los iones se adsorbe mejor sobre el adsorbente es decir mejor interacción ion - dipolo dejando de lado al adsorbato en este caso el mismo comportamiento se observa con los tres colorantes.

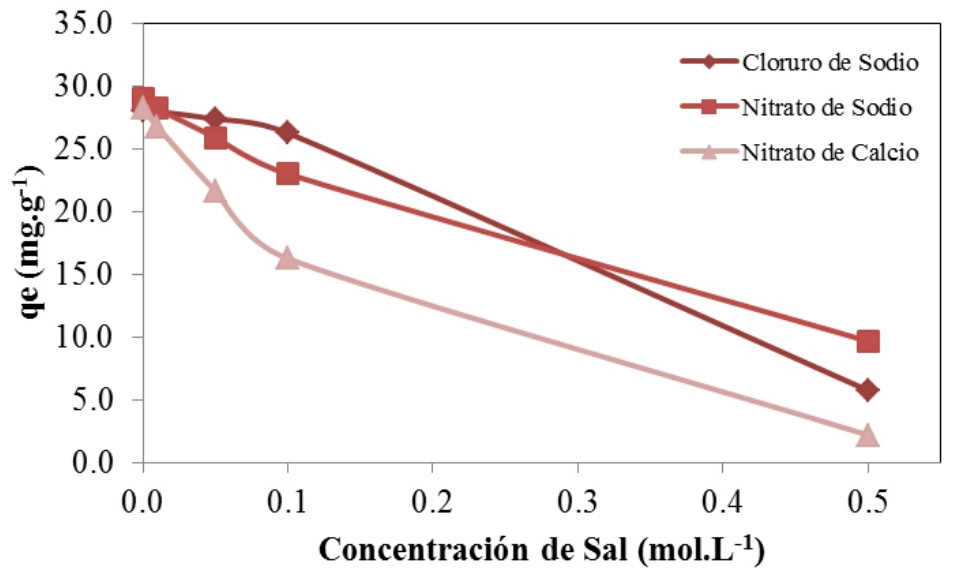

Figura 12. Efecto de la fuerza iónica en la adsorción de RA 18 con TV a $\mathrm{pH} 2$ y $\mathrm{T}$ ambiente

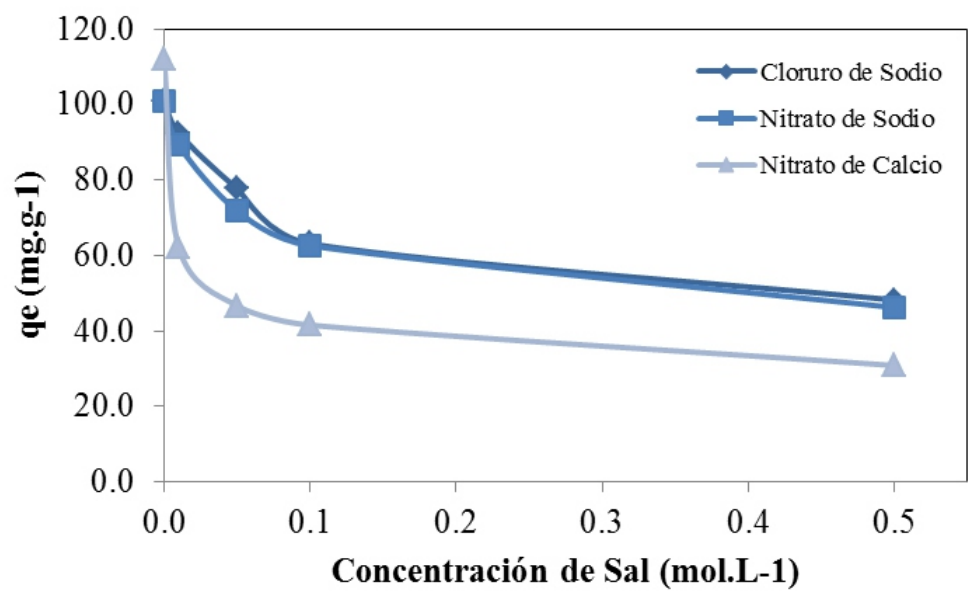

Figura 13. Efecto de la fuerza iónica en la adsorción de AZB 99 con $\mathrm{TV}$ a pH 4,5 y T ambiente 


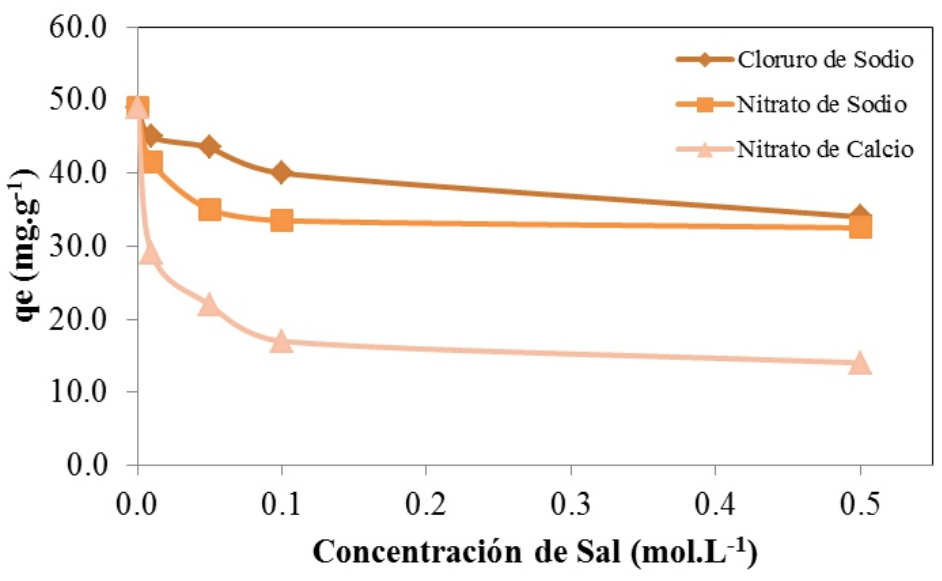

Figura 14. Efecto de la fuerza iónica en la adsorción de AM 57 con TV a $\mathrm{pH} 4$ y T ambiente

\section{CONCLUSIONES}

El adsorbente seleccionado, hojas molidas de desecho de té verde (TV) tiene la capacidad de adsorber los colorantes, con una buena eficiencia.

El adsorbente TV es mejor para el colorante azul y tiene una buena capacidad de adsorción y se ajusta a los modelos de Langmuir y Freundlich, y tiene una capacidad máxima de adsorción de $287,2 \mathrm{mg} \mathrm{g}^{-1}$.

La cantidad de adsorbente influye en la capacidad de adsorción; los resultados demuestran que con menor cantidad de adsorbente la adsorción es más eficiente.

El pH de la solución muestra un fuerte efecto en la capacidad de adsorción, siendo el óptimo para el colorante AM 57 de 4, para el AZB 99 de 4,5 y para el RA 18 de 2.

Altas concentraciones en la fuerza iónica disminuyen la capacidad de adsorción de los tres colorantes.

\section{AGRADECIMIENTOS}

Los autores quieren agradecer a la empresa L'Oreal Perú, por el financiamiento otorgado a través del premio "POR LA MUJER EN LA CIENCIA 2012" y al Consejo Nacional de Ciencia y Tecnología e Innovación Tecnología (CONCYTEC) del Perú. También hacen extensivo el agradecimiento a la Sección Química Dpto. de Ciencias Exactas Facultad de Ciencias y Filosofía Alberto Cazorla Talleri de la Universidad Peruana Cayetano Heredia y al Departamento de Ciencias Sección Química de la Facultad de Ciencias e Ingeniería de la Pontificia Universidad Católica del Perú, por las facilidades otorgadas para la realización de la investigación. 


\section{BIBLIOGRAFÍA}

1. G. Nohynek, R. Fautz, F. Benech-Keffer, H. Toutain. Food and Chem Toxicol. 2004; 42: 517-543.

2. W. Steiling, J. Kreutz, H. Hofer, Toxicol. In Vitro, 2001; 15: 565-570.

3. W. Steiling, ATLA 32, Supplement 1, 2004, 689-692.

4. R. Bronaugh, E. Congdon, J. Invest. Dermatol., 1984; 83: 124-127.

5. S. Mozia, M. Tomaszweska, A. Morawski, Desalination, 2005, 449-456.

6. Y. Chaisuksant, Environ. Technol., 2003; 24: 1501-1508.

7. M. Reátegui, H. Maldonado, M. Ly, E. Guibal, Adv. Mater. Res., 2009; 71-73: 585-588.

8. D. Park, S. Lim, Y. Yun, J. Park. Bioresour. Technol., 2008; 18: 8810-8818.

9. Scientific Committee on Cosmetic Products and Non-Food Products intended for consumers, COLIPAC1 (2003) y COLIPA C175 (2004).

10. F. Andersen. Internat. J. Toxicol. 2007; 26: 51-63.

11. N. Tapia. Adsorción y Biosorción, Mecanismos y principales modelos para describir el fenómeno de biosorción. Curso Aplicaciones ambientales de biotecnología en la industria, PUCP, Agosto 2002.

12. Q. Juihui, J. Environ. Sci., 2008, 20, 1-13.

13. M. Darder, P. Aranda, E. Ruiz-Hitzky, Adv. Mater., 2007; 19: 1309-1319.

14. T. Davis, B. Volesky, A. Mucci, Water. Res., 2003 ; 37: 4311-4330.

15. F. Xu, A. Bhandari, J. Agric. Food Chem., 2003; 51: 183-188.

16. E. Morris, D. Rees, D. Thorn, Carbohydrate Res., 1978; 66: 145-154.

17. Yu Liu and Jian log Wang, Fundamentals and Application of Biosorption Isotherms, Kinetic and thermodynamics, Ed. Envirimental and Technology Series 2009, 94. 